\title{
Patterns and Waves Generated by a Subcritical Instability in Systems with a Conservation Law under the Action of a Global Feedback Control
}

\author{
Y. Kanevsky * and A.A. Nepomnyashchy \\ Department of Mathematics, Technion - Israel Institute of Technology, \\ Haifa 32000, Israel
}

\begin{abstract}
A global feedback control of a system that exhibits a subcritical monotonic instability at a non-zero wavenumber (short-wave, or Turing instability) in the presence of a zero mode is investigated using a Ginzburg-Landau equation coupled to an equation for the zero mode. The method based on a variational principle is applied for the derivation of a low-dimensional evolution model. In the framework of this model the investigation of the system's dynamics and the linear and nonlinear stability analysis are carried out. The obtained results are compared with the results of direct numerical simulations of the original problem.
\end{abstract}

Key words: feedback control, pattern formation, variational approach

AMS subject classification: 35B36, 93B52

\section{Introduction}

Many pattern forming systems have symmetries or conservation laws that make their dynamics different from the one of "ordinary" pattern-forming systems [3, 4, 15, 16, 5]. Examples of such systems include Marangoni convection in a liquid layer with deformable interface heated from below [9, 10], instabilities in multimode lasers [1], nonlinear dynamics of sand banks and sand waves [13], Asaro-Tiller-Grinfeld instability of an epitaxial solid film in the presence of wetting interactions with the substrate [6], and many others (see [16]). The characteristic feature of such

*Corresponding author. E-mail: yuliya@techunix.technion.ac.il 
systems is that near the threshold of the short-wave instability, their dynamics is described by a system of coupled equations: for the unstable mode and for the slow Goldstone mode.

In the present paper, we consider systems subjected to a short-wave monotonic (Turing) instability and characterized by the conservation of a certain "mass" variable $[16,9,10,6]$. The generic system of amplitude equations is as follows:

$$
\begin{aligned}
& A_{t}=A+A_{x x}-\lambda|A|^{2} A+A B, \\
& B_{t}=m_{w} B_{x x}+w\left(|A|^{2}\right)_{x x},
\end{aligned}
$$

where $A$ is a complex amplitude of the unstable short-wave monotonic (Turing) mode and $B$ is the amplitude of the zero mode associated with the conservation of mass. Here $m_{w}>0$, while $w$ can be of either sign. Note that all the coefficients in (1.1) are real.

In a contradistinction to previous works on that subject, we consider here the case where the short-wave instability is subcritical, i.e., $\lambda=-1$ in (1.1). One could assume that in that case the weakly nonlinear amplitude equations (1.1) are of no use, because the solutions bifurcating subcritically are unstable, and a blow-up is unavoidable (recall that the coefficients in (1.1) are real, therefore the dispersion mechanism of stabilization $[20,1]$ does not work). However, these solutions can be stabilized by means of a feedback control. That stabilization can be considered as a tool for the numerical investigation of the set of bifurcating solutions in the subcritical region of parameters.

In the present paper, we apply a nonlinear control that affects only the linear growth rate of the primary instability. In order to avoid the blow-up that can develop in any spatial point, it is natural to measure and to control the maximum value of a characteristic variable (i.e., front deformation in the case of a morphological instability) over the whole region. Near the instability threshold, the deviation of that variable from its undisturbed value will be proportional to $\max _{x}|A|$. The simplest way of control, that we consider here, is making the deviation of the growth rate proportional to that quantity. In that case, we arrive to the following generic system of amplitude equations under global feedback control:

$$
\begin{aligned}
& A_{t}=\left(1-p \max _{x}|A|\right) A+A_{x x}+|A|^{2} A+A B, \\
& B_{t}=m_{w} B_{x x}+w|A|_{x x}^{2}
\end{aligned}
$$

where $p$ is the parameter characterizing the control strength. This way of control can be achieved by changing the global parameters of the system. For instance, in the case of a morphological instability of the solidification front it is implemented by changing the sample velocity and applied temperature gradient [17], in the case of a Marangoni instability it is sufficient to change the applied heat flux, etc. It has been shown formerly that this way of control is efficient for arresting a localized blow-up in a number of pattern-formation problem, including the Sivashinsky equation [17], the Ginzburg-Landau equation with real [19] and complex [8] coefficients, as well as for controlling patterns described by the generalized Swift-Hohenberg equation [23].

Recently, in [7] it has been shown that the above-mentioned type of a feedback control can stabilize the system (1.2) and lead to the formation of localized unipulse stationary states, or traveling solitary waves. It has been found that the unipulse traveling structures result from an instability of 
the stationary unipulse structures when the coupling parameter $w$ exceeds a critical value that is determined by the zero mode damping coefficient $m_{w}$.

The simple shape of pulses makes it attractive to use low-dimensional models for the description of their dynamics. There exist several methods of a reduction of an original equation to a finite set of ordinary differential equations (ODE). One of these methods utilizes the integrals of energy, momentum and a finite number of higher-order generalized moments, and it has been applied for the analysis of the dynamics of localized waves of a cubic-quintic complex Ginzburg-Landau equation (CGLE) [24]. Another method is based on a modified variational technique. In the case of the complex Ginzburg-Landau equation both methods lead to identical or similar models [11]. Low-dimensional (ODE) models, derived by means of a variational approach, have been widely used for solving problems of nonlinear optics governed primarily by partial differential equations [14], including non-conservative problems $[2,22]$. Recently, it has been demonstrated that lowdimensional models can reproduce details of the temporal dynamics of pulses under the action of an instantaneous [11] and delayed [12] feedback control in an oscillatory system governed by a subcritical complex Ginzburg-Landau equation. Let us note also that the dynamics of localized states (fronts) has been described by low-dimensional ODEs in the analysis of control in reactiondiffusion systems (see, e.g., [21]).

In the present paper, the coupled system (1.2) is used as the object for the application of the variational approach discussed above. In Sec. 2. we consider stationary localized solutions and investigate their stability; the transition to traveling-wave solutions is discussed. In Sec. 3. we derive a finite-dimensional dynamical model which is used for modeling the behavior of the system and for the linear stability analysis. In Sec. 4. the analytical results obtained in the framework of the derived ODE-model are compared with the results of a direct numerical simulation of the original equation; the conclusions are given.

\section{Analytical solutions}

\subsection{Stationary solutions}

In the present subsection we consider stationary solutions of the problem (1.2). The structures obtained in [7] by means of direct numerical simulation of (1.2) are characterized by a constant value of the phase $\arg (A)$. Therefore, we can fix that value equal to zero and arrive to the following system of equations for real variables $A$ and $B$ :

$$
\begin{gathered}
A_{x x}+\left(1-p \max _{x} A\right) A+A^{3}+A B=0, \\
m_{w} B_{x x}+w\left(A^{2}\right)_{x x}=0 ; \quad-\infty<x<\infty .
\end{gathered}
$$

The variable $B(x)$ is normalized in such a way that its averaged value over the whole domain is equal to zero,

$$
\langle B(x)\rangle=0 .
$$


Recall that $m_{w}>0$, while $w$ may have either sign.

The solution $B(x)$ of (2.2) bounded as $x \rightarrow \pm \infty$ and satisfying the condition (2.3) is:

$$
B(x)=\left(-\frac{w}{m_{w}}\right)\left(|A(x)|^{2}-\left\langle A^{2}\right\rangle\right),
$$

which leads to the following closed equation for the amplitude $A(x)$ :

$$
A_{x x}-\alpha A+\beta A^{3}=0,
$$

where

$$
\alpha=-1+p \max _{x}|A|+\frac{w}{m_{w}}\left\langle A^{2}\right\rangle, \beta=1-\frac{w}{m_{w}} .
$$

The coefficient $\alpha$ determines the effective linear decay rate of the short-wave amplitude in the presence of the global control and the zero mode, while $\beta$ is the Landau coefficient renormalized due to the action of the zero mode. The constants $\alpha$ and $\beta$ can have any signs.

Integration of (2.4) gives the relation

$$
\frac{A_{x}^{2}}{2}-\frac{\alpha A^{2}}{2}+\frac{\beta A^{4}}{4}=E_{A}=\text { const. }
$$

In the case $\alpha>0, \beta<0$ equation (2.6) has no bounded solutions except $A(x)=0$. In other cases relation (2.6) determines a set of closed orbits in the phase plane $\left(A, A_{x}\right)$ corresponding to spatially periodic solutions, as well as separatrices that describe pulse-like or front-like solutions, and fixed points corresponding to constant solutions.

In the case $\alpha>0, \beta>0$, there are two kinds of periodic solutions. When $-\alpha^{2} / 4 \beta<E_{A}<0$, the solutions are sign-preserving. Solutions with positive and negative $A$ are obtained from each other by a simple change of the sign and are equivalent in all the aspects; later on, we consider positive solutions. Solving (2.4) gives a family of solutions in terms of Jacobi elliptic functions,

$$
A(x)=A_{M} \mathrm{dn}\left[A_{M} \sqrt{\frac{\beta}{2}}\left(x-x_{0}\right) ; m\right],
$$

where $A_{M}$ is connected with the parameter $\alpha$ by the relation $\alpha=(2-m) \beta A_{M}^{2} / 2$, and $m$ is the modulus of the elliptic function. With $\left\langle A^{2}\right\rangle=A_{M}^{2} E(m) / K(m)$ equation (2.5) gives the following relation that determines the amplitude $A_{M}$ as a function of $m$ :

$$
\left[\frac{1}{2}\left(1-\frac{w}{m_{w}}\right)(2-m)+\frac{w}{2 m_{w}} \frac{E(m)}{K(m)}\right] A_{M}^{2}-p A_{M}+1=0 .
$$

The period of the solution (2.7) is

$$
L=\frac{2 \sqrt{2} K(m)}{A_{M} \sqrt{1-w / m_{w}}} .
$$


In the limit $m \rightarrow 1\left(E_{A} \rightarrow 0\right)$ a periodic solution tends to a solitary-wave solution such that both $A(x)$ and $B(x)$ tend to zero at infinity. In this case

$$
B(x)=-\frac{w}{m_{w}} A^{2},
$$

and the function $A(x)$ satisfies the equation

$$
A_{x x}+\left(1-p \max _{x} A\right) A+\left(1-\frac{w}{m_{w}}\right) A^{3}=0 .
$$

Two nonzero localized solutions of (2.8), $\left(A_{ \pm}(x), B_{ \pm}(x)\right)$ exist in the following interval of the coupling parameter $w$ :

$$
w_{\min }=m_{w}\left(2-p^{2}\right) / 2 \leq w<m_{w}
$$

and are given by

$$
A_{ \pm}(x)=a_{ \pm} \operatorname{sech}\left[\kappa_{ \pm}\left(x-x_{0}\right)\right], B_{ \pm}(x)=-\frac{w}{m_{w}} a_{ \pm}^{2} \operatorname{sech}^{2}\left[\kappa_{ \pm}\left(x-x_{0}\right)\right]
$$

where

$$
a_{ \pm}=\frac{p \pm \sqrt{p^{2}-2\left(1-w / m_{w}\right)}}{1-w / m_{w}}, \kappa_{ \pm}=\frac{1}{\sqrt{2}} \sqrt{1-\frac{w}{m_{w}}} a_{ \pm} .
$$

For $\alpha>0, \beta>0, E_{A}>0$ equation (2.6) has a family of solutions

$$
A(x)=A_{c} \mathrm{cn}\left[A_{c} \sqrt{\frac{\beta}{2 m}}\left(x-x_{0}\right) ; m\right]
$$

where

$$
A_{c}^{2}=\frac{\alpha}{\beta} \frac{2 m}{2 m-1}
$$

$1 / 2<m<1$. These periodic solutions are not sign-preserving: they have the property $A(x+$ $L / 2)=-A(x)$, where $L$ is the period. Note that $\alpha$, which is determined by (2.5), is a function of $A_{c}$ and $m$, therefore relation (2.12) is actually an equation for $A_{c}(m)$.

In the case $\alpha<0, \beta>0$, the periodic solutions, which exist for $E_{A}>0$, are determined by exactly the same formulas (2.11), (2.12), but with $0<m<1 / 2$.

Finally, in the case $\alpha<0, \beta<0$ (i.e., $w>m_{w}$ ), the periodic solutions, which exist for $0<E_{A}<-\alpha^{2} / 4 \beta$, are determined by the following formula:

$$
A(x)=A_{s} \operatorname{sn}\left[A_{s} \sqrt{\frac{-\beta}{2 m}}\left(x-x_{0}\right) ; m\right]
$$

where

$$
A_{s}^{2}=\frac{\alpha}{\beta} \frac{2 m}{1+m}, 0<m<1
$$


In the limit $m \rightarrow 1$ we obtain the solution

$$
A(x)=\sqrt{\frac{\alpha}{\beta}} \tanh \sqrt{-\frac{\alpha}{2}}\left(x-x_{0}\right)
$$

that corresponds to a "black hole" in a homogeneous short-wave pattern. For the amplitude $a^{2}=$ $\alpha / \beta$, the self-consistency equation

$$
1-p a+\left(1-\frac{w}{m_{w}}\right) a^{2}=0
$$

determines the single branch of solutions

$$
a=\frac{\sqrt{p^{2}+4\left(w / m_{w}-1\right)}-p}{2\left(w / m_{w}-1\right)}
$$

for any value of $p$. Note that $\alpha=-a^{2}$ and

$$
B=-\frac{w}{m_{w}} a^{2} \operatorname{sech}^{2} \frac{a}{\sqrt{2}}\left(x-x_{0}\right) .
$$

\subsection{Instability of sign-changing solutions}

The analysis carried out in the previous subsection reveals the existence of two qualitatively different types of stationary solutions: sign-preserving solutions (2.7), (2.9), and sign-changing solutions (2.11), (2.13). The latter type of solutions contains points where $A$ is exactly equal to zero, i.e., the amplitude $|A|$ vanishes and the phase $\arg (A)$ has a jump equal to $\pi$. In the present subsection, we show that all sign-changing stationary solutions are subject to a monotonic instability with respect to phase-changing disturbances that smoothen the phase jump. Recall that for a pure GinzburgLandau equation with real coefficients, a similar instability of solutions of the type (2.14) was first found by Newell and Whitehead [18].

Linearization of Eq. (1.2) around a real stationary solution $(A(x), B(x))$ leads to two separate systems of equations for real disturbances $\left(\tilde{A}^{r}, \tilde{B}\right)$ and imaginary disturbances $\left(i \tilde{A}^{i}, 0\right)$. For our goal, it is sufficient to consider imaginary disturbances governed by the following equation:

$$
\tilde{A}_{t}^{i}=\tilde{A}_{x x}^{i}-\alpha \tilde{A}^{i}+\beta A^{2} \tilde{A}^{i}
$$

Taking $\tilde{A}^{i}(x, t)=u(x) \exp (\sigma t)$, we obtain the eigenvalue problem

$$
u_{x x}+\left(\beta A^{2}-\alpha-\sigma\right) u=0,|u( \pm \infty)|<\infty .
$$

Obviously, problem (2.15) always has a solution $u=A, \sigma=0$, corresponding to a homogeneous phase change of the $A$-component of the stationary solution. If (2.15) has an eigenvalue $\sigma>0$, then the solution $(A, B)$ is unstable. 
For the base solution (2.11) $(\beta>0)$, we define $z=\sqrt{\beta /(2 m)} A_{c} x$ and obtain the following problem:

$$
u_{z z}+2 m\left(1-\frac{\alpha+\sigma}{\beta A_{c}^{2}}-\operatorname{sn}^{2} z\right) u=0,|u( \pm \infty)|<\infty .
$$

It can be checked directly that $u=\operatorname{dn} z$ is the eigenfunction of (2.16) with the eigenvalue

$$
\sigma=-\alpha+\frac{\beta A_{c}^{2}}{2}
$$

Substituting (2.12), we find that

$$
\sigma=\frac{\alpha(1-m)}{2 m-1}
$$

Taking into account that $1 / 2<m<1$ for $\alpha>0$ and $0<m<1 / 2$ for $\alpha<0$, we conclude that $\sigma>0$ in both cases. Thus, solutions (2.11) are unstable.

For the base solution (2.13) $(\beta<0, \alpha<0)$, we define $z=\sqrt{-\beta /(2 m)} A_{s} x$ and arrive at the problem

$$
u_{z z}+2 m\left(\frac{\alpha+\sigma}{\beta A_{s}^{2}}-\operatorname{sn}^{2} z\right) u=0,|u( \pm \infty)|<\infty .
$$

Again, $u=\operatorname{dn} z$ is the eigenfunction with

$$
\sigma=-\frac{\alpha}{1+m}>0
$$

Thus, solutions (2.13) are unstable.

We conclude that all the sign-changing stationary solutions are unstable.

\subsection{Stability of localized pulses}

Now we consider the stability of localized stationary solutions (2.9) and (2.10). In the absence of zero mode the stability of these solutions has been studied in [19]. In that case, for arbitrary values of parameters the upper branch is unstable with respect to real (amplitude) disturbances, while the lower branch is stable. Here we obtain a similar result in the presence of zero mode near the point

$$
p=p_{*}=\sqrt{2 \beta}, \beta=1-\frac{w}{m_{w}}
$$

where two branches of stationary solutions are born due to a saddle-node bifurcation.

Let us parameterize the family of solutions (2.9), (2.10) using parameter $k$. Take $x_{0}=0$ (obviously, the stability does not depend on $x_{0}$ ). Both branches of solutions can be written as

$$
A=a \cosh ^{-1} k x, B=-\frac{w}{m_{w}} A^{2},
$$

where

$$
a=\sqrt{\frac{2}{\beta}} k, p=\sqrt{\frac{\beta}{2}} \frac{k^{2}+1}{k} .
$$


Solutions with $k<1$ correspond to the lower branch, while solutions with $k>1$ correspond to the upper branch.

The time evolution of real disturbances $\left(\tilde{A}^{r}, \tilde{B}\right)$ on the background of $(2.17)$ is governed by the following system of functional-differential equations:

$$
\begin{gathered}
\tilde{A}_{t}^{r}=\tilde{A}_{x x}^{r}+\left(-k^{2}+3 A^{2}+B\right) \tilde{A}^{r}+A \tilde{B}-p A \tilde{A}^{r}(0, t), \\
\tilde{B}_{t}=m_{w} \tilde{B}_{x x}+2 w\left(A \tilde{A}^{r}\right)_{x x} .
\end{gathered}
$$

Defining $z=k x$ and assuming $\tilde{A}^{r}=u(z) \exp (\sigma t), \tilde{B}=v(z) \exp (\sigma t)$, we obtain the following system:

$$
\begin{gathered}
u_{z z}(z)+\left[-1-\frac{\sigma}{k^{2}}+\frac{2(2+\beta)}{\beta \cosh ^{2} z}\right] u(z)+\frac{\sqrt{2}}{k \sqrt{\beta}} \frac{v(z)}{\cosh z}=\left(1+\frac{1}{k^{2}}\right) \frac{u(0)}{\cosh z}, \\
v_{z z}(z)+\frac{2 w}{m_{w}} \sqrt{\frac{2}{\beta}} k\left[\frac{u(z)}{\cosh z}\right]_{z z}-\frac{\sigma}{m_{w} k^{2}} v(z)=0 .
\end{gathered}
$$

Due to the symmetry of the problem, both components of the eigenfunction $(u(z), v(z))$ are either even or odd. For odd eigenfunctions, $u(0)=0$, while for even ones generally $u(0) \neq 0$. In the present section, we consider even disturbances and normalize the solution of problem (2.18), (2.19) by the condition

$$
u(0)=1 .
$$

For $k=1$, i.e. in the point of the saddle-node bifurcation leading to the creation of two branches of solutions for $p>\sqrt{2 \beta}$, problem (2.18), (2.19) has an exact solution,

$$
\sigma=0, u=u_{0}=\frac{1}{\cosh z}-z \sinh z \cosh ^{2} z, v=v_{0}=-\frac{2 w}{m_{w}} \sqrt{\frac{2}{\beta}} \frac{u_{0}}{\cosh z} .
$$

Near that point, we apply the expansion

$$
\frac{1}{k^{2}}=1+\varepsilon, \sigma=\varepsilon \sigma_{1}+\ldots, u=u_{0}+\varepsilon u_{1}+\ldots, v=v_{0}+\varepsilon v_{1}+\ldots
$$

Note that $k=1-\varepsilon / 2+\ldots$, hence $\varepsilon>0(\varepsilon<0)$ corresponds to the lower (upper) branch of solutions.

At the first order in $\varepsilon$, the following system is obtained:

$$
\begin{gathered}
\left(u_{1}\right)_{z z}+\left[\frac{2(2+\beta)}{\beta \cosh ^{2} z}-1\right] u_{1}+\sqrt{\frac{2}{\beta}} \frac{v_{1}}{\cosh z}=\frac{1}{\cosh z}+\sigma_{1} u_{0}-\frac{1}{2} \sqrt{\frac{2}{\beta}} \frac{v_{0}}{\cosh z}, \\
\left(v_{1}\right)_{z z}+\frac{2 w}{m_{w}} \sqrt{\frac{2}{\beta}}\left(\frac{u_{1}}{\cosh z}\right)_{z z}=\frac{\sigma_{1}}{m_{w}} v_{0}+\frac{w}{m_{w}} \sqrt{\frac{2}{\beta}}\left(\frac{u_{0}}{\cosh z}\right)_{z z} .
\end{gathered}
$$

Eliminating $v_{1}$, we find:

$$
\left(u_{1}\right)_{z z}+\left(-1+\frac{6}{\cosh ^{2} z}\right) u_{1}=\frac{1}{\cosh z}+\sigma_{1}\left[u_{0}+\frac{2}{\beta} \frac{w}{d^{2}} \frac{z \sinh z}{\cosh ^{2} z}\right]
$$




$$
\left|u_{1}\right|<\infty, z \rightarrow \pm \infty .
$$

Problem (2.20) is solvable when its right-hand side is orthogonal to the solution of the homogeneous problem, which is $u_{0}$, hence

$$
\sigma_{1}=-\frac{\int_{-\infty}^{\infty} u_{0} \operatorname{sech} z d z}{\int_{-\infty}^{\infty} u_{0}\left[u_{0}+\frac{2}{\beta} \frac{w}{m_{w}^{2}} \frac{z \sinh z}{\cosh ^{2} z}\right] d z}
$$

Calculating the integral, we find:

$$
\sigma_{1}=-\frac{18}{\left(12+\pi^{2}\right)-2\left(\pi^{2}-6\right) w /\left(\beta m_{w}^{2}\right)} .
$$

One can see that if

$$
w<w_{*}=\frac{m_{w}^{2}}{m_{w}+2\left(\pi^{2}-6\right) /\left(\pi^{2}+12\right)},
$$

then $\sigma_{1}<0$. That means that in the region (2.21) the upper branch (that with $\varepsilon<0$ ) is unstable, while the lower branch (that with $\varepsilon>0$ ) is stable with respect to even real disturbances. In [7] it was shown that the stationary solutions become unstable with respect to odd disturbances leading to the development of traveling waves at the value $w=w_{0}=m_{w}^{2} /\left(m_{w}+2\right)<w_{*}$ (see also Sec. 2.4.). Therefore, there is no need in the consideration of the stability of stationary solutions in the region $w>w_{*}$.

\subsection{Traveling waves}

In the present section we recall briefly the main results on the bifurcation of traveling-wave solutions obtained in [7]. These results will be used for the construction of a low-dimensional model in Sec. 3.

Numerical simulations in [7] reveal the transition of stationary solutions to traveling wave solutions. For $w>w_{0}$, a transition to either a traveling wave moving to the right,

$$
A(x, t)=A_{r}\left(X_{r}\right), B(x, t)=B_{r}\left(X_{r}\right), X_{r}=x-\mathrm{v} t, \mathrm{v}>0,
$$

or a traveling wave moving to the left,

$$
A(x, t)=A_{l}\left(X_{l}\right), B(x, t)=B_{l}\left(X_{l}\right), X_{l}=x+\mathrm{v} t, \mathrm{v}>0,
$$

is observed. Due to the symmetry of the problem, these two solutions are obtained from each other by reflection $x \rightarrow-x$, and their properties are equivalent. Later on. we consider waves (2.22) using the notation $X_{r}=X$.

The traveling wave solution satisfies the system of equations

$$
\begin{gathered}
A_{X X}+\mathrm{v} A_{X}+A\left(1-p \max _{X}|A|\right)+A^{3}+A B, \\
m_{w} B_{X X}+\mathrm{v} B_{X}+w\left(A^{2}\right)_{X X}=0
\end{gathered}
$$


with periodicity conditions

$$
A(X+L)=A(X), B(X+L)=B(X)
$$

and the condition of zero mean value of $B$ :

$$
\langle B\rangle=\frac{1}{L} \int_{-L / 2}^{L / 2} B(X) d X=0 .
$$

The nonlinear analysis of the bifurcation has been performed in the limit $L \gg 1$, which corresponds to the numerical simulations. In this case the elliptic function (2.7) can be approximated by the solitary-wave solution (2.9). The following expression for the threshold of the transition from the stationary to traveling-wave solutions is derived:

$$
w_{0}=\frac{m_{w}^{2}}{m_{w}+2} .
$$

The expansions utilized in the bifurcation analysis,

$$
\begin{aligned}
A(X) & =A_{0}+\varepsilon A_{1}+\varepsilon^{2} A_{2}+\ldots \\
B(X) & =B_{0}+\varepsilon B_{1}+\varepsilon^{2} B_{2}+\ldots \\
\mathrm{v} & =\varepsilon \mathrm{v}_{1}+\ldots, \quad w=w_{0}+\varepsilon^{2} w_{2}+\ldots
\end{aligned}
$$

lead to the following asymptotic expansion of the solution:

$$
\begin{aligned}
A(X)= & \frac{a}{\cosh (\kappa X)}+\varepsilon O(1 / L)+o(\varepsilon), \\
B(X)= & -\frac{w_{0} a^{2}}{m_{w}}\left(\frac{1}{\cosh ^{2}(\kappa X)}-\frac{2}{\kappa L}\right) \\
& +\varepsilon \frac{\mathrm{v}_{1} w_{0} a^{2}}{m_{w}^{2} \kappa}\left(\tanh (\kappa X)-\frac{2 X}{L}\right)+o(\varepsilon) .
\end{aligned}
$$

It has been also found that in the threshold point there holds

$$
\frac{d \mathrm{v}^{2}(w)}{d w}=\frac{\mathrm{v}_{1}^{2}}{w_{2}}=\frac{2 \kappa\left(m_{w}+2\right)^{2}}{L} .
$$

This quantity tends to zero as $L \rightarrow \infty$.

\section{Variational model}

In the present section we construct a low-dimensional model for studying the dynamics of system of equations (2.1) and (2.2). For this purpose we utilize a modified variational approach that has been formerly applied in $[2,22,11,12]$. 
Following [7] we define the operator

$$
\partial_{x}^{-1} B=\int_{0}^{x} B(y) d y-\left\langle\int_{0}^{x} B(y) d y\right\rangle
$$

and rewrite the system $(2.1),(2.2)$ in the form

$$
\begin{aligned}
A+A_{x x}+A^{3}+A B & =A_{t}+p\left(\max _{x} A\right) A \equiv Q_{1}, \\
\frac{m_{w}}{2 w} B+\frac{1}{2} A^{2} & =\frac{1}{2 w} \partial_{x}^{-1} B_{t} \equiv Q_{2} .
\end{aligned}
$$

In the absence of terms $Q_{1}$ and $Q_{2}$, equations (3.1), (3.2) would be the Euler-Lagrange equations for the functional

$$
S=-\int_{-L / 2}^{L / 2} \mathcal{L}[A, B] d x
$$

with

$$
\mathcal{L}[A, B]=-\frac{1}{2} A^{2}-\frac{1}{4} A^{4}+\frac{1}{2} A_{x}^{2}-\frac{m_{w}}{4 w} B^{2}-\frac{1}{2} A^{2} B .
$$

Thus, there holds:

$$
\frac{\delta S}{\delta A}=Q_{1} \quad \text { and } \quad \frac{\delta S}{\delta B}=Q_{2} .
$$

A modified variational approach for the system of equations (3.1), (3.2) with an ansatz $A(x, t)=A\left(b_{1}(t), \ldots, b_{N}(t), x\right), B(x, t)=B\left(b_{1}(t), \ldots, b_{N}(t), x\right)$, leads to the following system of equations:

$$
-\frac{\partial<\mathcal{L}>}{\partial b_{j}}=\int_{-L / 2}^{L / 2} Q_{1} \frac{\partial A}{\partial b_{j}} d x+\int_{-L / 2}^{L / 2} Q_{2} \frac{\partial B}{\partial b_{j}} d x, \quad j=1, \ldots, N
$$

where

$$
<\mathcal{L}>=\int_{-L / 2}^{L / 2} \mathcal{L}\left[A\left(b_{1}(t), b_{2}(t), \ldots, b_{N}(t), x\right), B\left(b_{1}(t), b_{2}(t), \ldots, b_{N}(t), x\right)\right] d x .
$$

The derivation is given in the Appendix.

For studying the dynamics of a traveling-wave solution, we use the ansatz compatible with the asymptotic expansion (2.24), (2.25),

$$
\begin{aligned}
A= & \frac{a_{w}(t)}{\cosh [\kappa(t)(x-s(t))]} \\
B= & b_{w}(t)\left[\frac{1}{\cosh ^{2}[\kappa(t)(x-s(t))]}-\frac{2}{\kappa L}\right] \\
& +c_{w}(t)\left[\tanh [\kappa(t)(x-s(t))]-\frac{2(x-s(t))}{L}\right],
\end{aligned}
$$


with $a_{w}(t), b_{w}(t), c_{w}(t), \kappa(t)$ and $s(t)$ playing the role of $b_{1}(t), \ldots, b_{5}(t)$ and with $x$ in the interval $(-L / 2, L / 2)$. In the computation of integrals the leading order in the limit $L \gg 1$ was considered.

The obtained evolution equations for $a_{w}(t), b_{w}(t), c_{w}(t), \kappa(t)$ and $s(t)$ are as follows:

$$
\begin{aligned}
\left(1+\pi^{2} / 3\right) w a_{w}^{2} \kappa_{t} & =2 b_{w} \kappa\left(m_{w} b_{w}+w a_{w}^{2}\right)-2 w a_{w}^{2} \kappa\left(2 \kappa^{2}-a_{w}^{2}-b_{w}\right), \\
6 \kappa\left(a_{w}\right)_{t}-3 a_{w} \kappa_{t} & =2 a_{w} \kappa\left(3-3 p a_{w}+2 a_{w}^{2}+2 b_{w}-\kappa^{2}\right), \\
L\left(b_{w} \kappa_{t}-\kappa\left(b_{w}\right)_{t}+c_{w} \kappa^{2} s_{t}\right) & =4 \kappa^{2}\left[m_{w} b_{w}+w a_{w}^{2}\right], \\
\frac{L^{2} \kappa\left(c_{w}\right)_{t}}{60}+b_{w} s_{t} & =-m_{w} \kappa c_{w}, \\
L\left(b_{w} \kappa\left(c_{w}\right)_{t}-c_{w} \kappa\left(b_{w}\right)_{t}+\right. & \left.b_{w} c_{w} \kappa_{t}\right)+\left(4 b_{w}^{2}-4 w a_{w}^{2} \kappa^{2}+L c_{w}^{2} \kappa\right) \kappa s_{t}=0 .
\end{aligned}
$$

The stationary localized solution of system (3.4)-(3.8) is obtained by substituting $\left(a_{w}\right)_{t}=$ $\left(b_{w}\right)_{t}=\left(c_{w}\right)_{t}=\kappa_{t}=s=0$. The exact solitary-wave solution (2.9), (2.10) is reproduced:

$$
\begin{aligned}
& a_{w}=\frac{p \pm \sqrt{p^{2}-2\left(1-w / m_{w}\right)}}{1-w / m_{w}}, \quad \kappa^{2}=\frac{1}{2}\left(1-w / m_{w}\right) a_{w}^{2} \\
& b_{w}=-\frac{w}{m_{w}} a_{w}^{2}, \quad c_{w}=0 .
\end{aligned}
$$

\subsection{Hopf bifurcation}

For a study of the Hopf bifurcation we consider the following expansions:

$$
\begin{aligned}
a_{w} & =a_{w, 0}+\varepsilon a_{w, 1}+\varepsilon^{2} a_{w, 2}+\ldots, \kappa=\kappa_{0}+\varepsilon \kappa_{1}+\varepsilon^{2} \kappa_{2}+\ldots, \\
b_{w} & =b_{w, 0}+\varepsilon b_{w, 1}+\varepsilon^{2} b_{w, 2}+\ldots, c_{w}=\varepsilon c_{w, 1}+\varepsilon^{2} c_{w, 2}+\ldots, \\
s_{t} & =\mathrm{v}=\varepsilon \mathrm{v}_{1}+\varepsilon^{2} \mathrm{v}_{2}+\ldots, \quad w=w_{0}+\varepsilon w_{1}+\varepsilon^{2} w_{2}+\ldots
\end{aligned}
$$

where the subscript index 0 denotes the stationary localized solution with $a_{w, 0}, b_{w, 0}$ and $\kappa_{0}$ given by (3.9). We substitute (3.10) into system (3.4)-(3.8), and after some algebraic transformation we obtain in the first order of $\varepsilon$ :

$$
\begin{aligned}
a_{w, 1} & =\frac{a_{w, 0}^{3}}{2 m_{w}\left(\kappa_{0}^{2}-1\right)} w_{1} \\
b_{w, 1} & =-\frac{1}{m_{w}}\left(2 a_{w, 0} w_{0} a_{w, 1}+a_{w, 0}^{2} w_{1}\right) \\
\kappa_{1} & =\frac{1}{4 \kappa_{0}}\left(2 a_{w, 0} a_{w, 1}+b_{w, 1}\right) \\
c_{w, 1} & =\kappa_{0} \mathrm{v}_{1} \\
w_{0} & =\frac{m_{w}^{2}}{m_{w}+2}
\end{aligned}
$$


The relation (3.15) coincides with the theoretical result (2.23) from [7].

The second order of $\varepsilon$ in (3.7), (3.8) leads to

$$
\begin{aligned}
c_{w, 2} & =\kappa_{0} \mathrm{v}_{2}, \\
w_{1} & =0,
\end{aligned}
$$

thus, it holds that $a_{w, 1}=\kappa_{1}=b_{w, 1}=0$. Therefore, from (3.4)-(3.6) we obtain the following:

$$
\begin{aligned}
a_{w, 2} & =\frac{a_{w, 0}}{6 m_{w}\left(\kappa_{0}^{2}-1\right)}\left(3 a_{w, 0}^{2} w_{2}-\kappa_{0} \mathrm{v}_{1}^{2} L\right), \\
b_{w, 2} & =-\frac{1}{4 m_{w}}\left(8 a_{w, 0} w_{0} a_{w, 2}+4 a_{w, 0}^{2} w_{2}-\kappa_{0} \mathrm{v}_{1}^{2} L\right), \\
\kappa_{2} & =\frac{1}{16 m_{w} \kappa_{0}}\left(8 m_{w} a_{w, 0} a_{w, 1}+4 m_{w} b_{w, 1}-\kappa_{0} \mathrm{v}_{1}^{2} L\right) .
\end{aligned}
$$

Equations (3.16)-(3.18) must be balanced, hence it is obvious that $\mathrm{v}_{1}^{2} \sim 1 / L$.

In the third order of $\varepsilon$ from (3.8) we derive

$$
8 b_{w, 0} b_{w, 2}-4 a_{w, 0}^{2} \kappa_{0}^{2} w_{2}-8 w_{0}\left(a_{w, 0}^{2} \kappa_{0} \kappa_{2}-\kappa_{0}^{2} a_{w, 0} a_{w, 2}\right)+\kappa_{0} c_{w, 1}^{2} L=0
$$

which leads to

$$
\frac{\mathrm{v}_{1}^{2}}{w_{2}}=\frac{2\left(m_{w}+2\right)^{2} \kappa_{0}}{L} .
$$

The latter result confirms the analytical result (2.26) from [7].

\subsection{Stability of a traveling-wave solution}

In the previous subsection we have seen that $\mathrm{v}_{1} \sim c_{w, 1} \sim 1 / \sqrt{L}$. Hence, in order to study the stationary traveling-wave solution, we consider $a_{w}, b_{w}, \kappa=O(1)$ and set $c_{w}=C_{w} / \sqrt{L}, s_{t}=\mathrm{v}=$ $\mathrm{V} / \sqrt{L}$. In this case system (3.4)-(3.8) is reduced to the following equations:

$$
\begin{aligned}
3-3 p a_{w}+2 a_{w}^{2}+2 b_{w}-\kappa^{2} & =0 \\
w a_{w}^{2}\left(2 \kappa^{2}-a_{w}^{2}-b_{w}\right)-b_{w}\left(m_{w} b_{w}+w a_{w}^{2}\right) & =0 \\
m_{w} b_{w}+w a_{w}^{2} & =\frac{C_{w} \mathrm{~V}}{4} \\
b_{w} \mathrm{~V} & =-m_{w} \kappa C_{w} \\
4 b_{w}^{2}-4 w a_{w}^{2} \kappa^{2}+C_{w}^{2} \kappa & =0
\end{aligned}
$$

From (3.21)-(3.23) it follows:

$$
m_{w} b_{w}^{2}+w a_{w}^{2} b_{w}=\frac{C_{w}}{4} b_{w} \mathrm{~V}=-\frac{m_{w}}{4} C_{w}^{2} \kappa=-\frac{m_{w}}{4}\left[-4 b_{w}^{2}+4 w a_{w}^{2} \kappa^{2}\right]
$$


therefore,

$$
b_{w}=-m_{w} \kappa^{2} .
$$

Using (3.24), we can rewrite eqs. (3.19) and (3.20) in the following form:

$$
\begin{aligned}
3-3 p a_{w}+2 a_{w}^{2} & =\left(1+2 m_{w}\right) \kappa^{2}, \\
m_{w}^{3} \kappa^{4}-2\left(1+m_{w}\right) w a_{w}^{2} \kappa^{2}+w a_{w}^{4} & =0 .
\end{aligned}
$$

Thus,

$$
\begin{aligned}
b_{w} & =-m_{w} \kappa^{2} \\
\mathrm{~V} & =\frac{C_{w}}{\kappa} \\
C_{w}^{2} & =4\left(w a_{w}^{2} \kappa-m_{w}^{2} \kappa^{3}\right) \\
\kappa^{2} & =\frac{a_{w}^{2}}{m_{w}^{3}}\left[w\left(1+m_{w}\right)-\sqrt{\left(1+m_{w}\right)^{2} w^{2}-w m_{w}^{3}}\right] \\
a_{w} & =\frac{3 p \pm \sqrt{9 p^{2}-12\left(2-\frac{1+2 m_{w}}{m_{w}^{3}}\left[w\left(1+m_{w}\right)-\sqrt{\left(1+m_{w}\right)^{2} w^{2}-w m_{w}^{3}}\right]\right)}}{2\left(2-\frac{1+2 m_{w}}{m_{w}^{3}}\left[w\left(1+m_{w}\right)-\sqrt{\left(1+m_{w}\right)^{2} w^{2}-w m_{w}^{3}}\right]\right)}
\end{aligned}
$$

The existence condition for a traveling-wave solution is $C_{w}^{2}>0$. From (3.27) it follows that the existence condition is equivalent to a condition $w>w_{0}$. If the denominator in (3.29) is negative then $a_{w}^{-}>0$, and if the denominator in (3.29) is positive then $a_{w}^{ \pm}>0$ under a certain condition on $p\left(p>2 / \sqrt{2+m_{w}}\right.$ is a sufficient condition for all $\left.w>w_{0}\right)$.

The validity of relation (3.29) is checked with substitution of $w=w_{0}$. For $w=w_{0}$ we obtain:

$$
\begin{aligned}
& \frac{w_{0}}{m_{w}^{3}}\left[\left(1+m_{w}\right)-\sqrt{\left(1+m_{w}\right)^{2}-m_{w}^{3} / w_{0}}\right]=\frac{1}{m_{w}+2}, \\
a_{w}^{-}= & \frac{3 p-3 \sqrt{p^{2}-4 / 3\left(2-\frac{1+2 m_{w}}{m_{w}+2}\right)}}{2\left(2-\frac{1+2 m_{w}}{m_{w}+2}\right)}=3 \frac{p-\sqrt{p^{2}-4 / 3 \frac{2 m_{w}+4-1-2 m_{w}}{m_{w}+2}}}{2 \frac{2 m_{w}+4-1-2 m_{w}}{m_{w}+2}} \\
= & \frac{p-\sqrt{p^{2}-4 \frac{1}{m_{w}+2}}}{2 \frac{1}{m_{w}+2}}
\end{aligned}
$$

which coincides with localized pulse solution (2.10) with $w$ taken equal to $w_{0}$.

For a stability test we consider a perturbed solution $a_{w}(t)=a_{w}+\tilde{a}_{w}, b_{w}(t)=b_{w}+\tilde{b}_{w}$, $\kappa(t)=\kappa+\tilde{\kappa}, C_{w}(t)=C_{w}+\tilde{C}_{w}$ and $\mathrm{V}(t)=\mathrm{V}+\tilde{\mathrm{V}}$, and linearize system (3.4)-(3.8):

$$
\begin{aligned}
\left(1+\pi^{2} / 3\right) w a_{w}^{2} \tilde{\kappa}_{t}= & 2 b_{w} \kappa\left(m_{w} \tilde{b}_{w}+2 w a_{w} \tilde{a}_{w}\right)+2\left(m_{w} b_{w}+w a_{w}^{2}\right)\left(b_{w} \tilde{\kappa}+\kappa \tilde{b}_{w}\right) \\
& -2 w a_{w}^{2} \kappa\left(4 \kappa \tilde{\kappa}-2 a_{w} \tilde{a}_{w}-\tilde{b}_{w}\right) \\
& -2 w\left(2 \kappa^{2}-a_{w}^{2}-b_{w}\right)\left(a^{2} \tilde{\kappa}+2 a_{w} \kappa \tilde{a}_{w}\right) \\
6 \kappa\left(\tilde{a}_{w}\right)_{t}-3 a_{w} \tilde{\kappa}_{t}= & 2 a_{w} \kappa\left(-3 p \tilde{a}_{w}+4 a_{w} \tilde{a}_{w}+2 \tilde{b}_{w}-2 \kappa \tilde{\kappa}\right)
\end{aligned}
$$




$$
\begin{aligned}
-L\left(\kappa\left(\tilde{b}_{w}\right)_{t}-b_{w} \tilde{\kappa}_{t}\right)+\left(\mathrm{V} \tilde{C}_{w}+C_{w} \tilde{\mathrm{V}}\right) \kappa^{2}= & 4 \kappa^{2}\left[m_{w} \tilde{b}_{w}+2 w a_{w} \tilde{a}_{w}\right] \\
b_{w} \tilde{\mathrm{V}}+\mathrm{V} \tilde{b}_{w}+\frac{L^{2} \kappa\left(\tilde{C}_{w}\right)_{t}}{60}= & -m_{w}\left(\kappa \tilde{C}_{w}+C_{w} \tilde{\kappa}\right) \\
L\left(b_{w} \kappa\left(\tilde{C}_{w}\right)_{t}-C_{w} \kappa\left(\tilde{b}_{w}\right)_{t}+b_{w} C_{w} \tilde{\kappa}_{t}\right)= & -\left(8 b_{w} \tilde{b}_{w}-8 w a_{w} \kappa\left(a_{w} \tilde{\kappa}+\kappa \tilde{a}_{w}\right)\right. \\
& \left.+C_{w}^{2} \tilde{\kappa}+2 C_{w} \kappa \tilde{C}_{w}\right) \kappa \mathrm{V}
\end{aligned}
$$

From (3.33)-(3.35) we obtain:

$$
\begin{array}{r}
\left.b_{w} \tilde{\kappa}_{t}-\kappa\left(\tilde{b}_{w}\right)_{t}=\frac{\kappa^{2}}{L}\left[4\left(m_{w} \tilde{b}_{w}+2 w a_{w} \tilde{a}_{w}\right)-\mathrm{V} \tilde{C}_{w}-C_{w} \tilde{\mathrm{V}}\right]\right) \\
\left(\tilde{C}_{w}\right)_{t}=\frac{60}{\kappa L^{2}}\left[-m_{w} \kappa \tilde{C}_{w}-m_{w} C_{w} \tilde{\kappa}-b_{w} \tilde{\mathrm{V}}-\mathrm{V} \tilde{b}_{w}\right] \\
4 b_{w} \tilde{b}_{w}-8 w a_{w}^{2} \kappa \tilde{\kappa}+C_{w}^{2} \tilde{\kappa}+C_{w} \kappa \tilde{C}_{w}-\kappa^{2} C_{w} \tilde{\mathrm{V}}=O(1 / L)
\end{array}
$$

From (3.36) it follows $\tilde{b}_{w}=-m_{w} \kappa \tilde{\kappa}$, thus the characteristic equation for the growth rate $\lambda$ is:

$$
\lambda^{2}+a_{1} \lambda+a_{0}=0
$$

where

$$
\begin{aligned}
& a_{1}=\frac{1}{3+\pi^{2}}\left[12 \kappa^{2}-\left(1+\frac{\pi^{2}}{3}\right)\left(4 a_{w}-3 p\right)\right] \\
& a_{0}=\frac{4}{3+\pi^{2}}\left[-a_{w}\left(4 a_{w}-3 p\right)\left(a_{w}^{2}+b_{w}\right)+4\left(1+m_{w}\right) \kappa^{2}\left(a_{w}^{2}+b_{w}-\kappa^{2}\right)\right] .
\end{aligned}
$$

A computer test of (3.39) shows that for $p \geq \sqrt{2}$ the solution with $a_{w}=a_{w}^{-}$exists and is stable.

\subsection{Limit of large $w$}

For $w \rightarrow \infty$ we can use the following expansion in a small parameter $1 / w$ :

$$
\begin{aligned}
\sqrt{w^{2}\left(1+m_{w}\right)^{2}-w m_{w}^{3}} & =w\left(1+m_{w}\right) \sqrt{1-\frac{m_{w}^{3}}{w\left(1+m_{w}\right)^{2}}} \\
& =w\left(1+m_{w}\right)\left[1-\frac{m_{w}^{3}}{2 w\left(1+m_{w}\right)^{2}}+o\left(\frac{1}{w^{2}}\right)\right]
\end{aligned}
$$

Applying (3.40) to (3.25)-(3.29), we obtain 


$$
\begin{aligned}
a_{w} & =\frac{1+m_{w}}{2 m_{w}+3}\left[3 p \pm \sqrt{9 p^{2}-6 \frac{3+2 m_{w}}{1+m_{w}}}\right] \\
\kappa^{2} & =\frac{a_{w}^{2}}{2\left(1+m_{w}\right)} \\
b_{w} & =-\frac{m_{w} a_{w}^{2}}{2\left(1+m_{w}\right)} \\
C_{w}^{2} & =4 \kappa a_{w}^{2} w \\
\mathrm{~V} & =\frac{C_{w}}{\kappa} .
\end{aligned}
$$

For $a_{w}=a_{w}^{+}$it holds:

$$
\begin{aligned}
a_{0}= & \frac{4 a_{w}^{3}}{3+\pi^{2}}\left[a_{w}-\left(4 a_{w}-3 p\right)\left(2+m_{w}\right) /\left(2+2 m_{w}\right)\right] \\
& =\frac{4 a_{w}^{3}}{2\left(3+\pi^{2}\right)\left(1+m_{w}\right)}\left[3 p\left(2+m_{w}\right)-a_{w}\left(2 m_{w}+6\right)\right] \\
& =\frac{4 a_{w}^{3}}{2\left(3+\pi^{2}\right)\left(1+m_{w}\right)\left(2 m_{w}+3\right)} \times \\
& {\left[-3 m_{w} p-\left(2 m_{w}+6\right)\left(1+m_{w}\right) \sqrt{9 p^{2}-6\left(2 m_{w}+3\right) /\left(1+m_{w}\right)}\right]<0, }
\end{aligned}
$$

therefore at least one eigenvalue is positive, thus a solution with $a_{w}=a_{w}^{+}$is unstable.

For $a_{w}=a_{w}^{-}$it follows:

$$
\begin{aligned}
a_{0}= & \frac{4 a_{w}^{3}}{3+\pi^{2}}\left[a_{w}-\left(4 a_{w}-3 p\right)\left(2+m_{w}\right) /\left(2+2 m_{w}\right)\right] \\
& =\frac{4 a_{w}^{3}}{2\left(3+\pi^{2}\right)\left(1+m_{w}\right)\left(2 m_{w}+3\right)} \times \\
& {\left[-3 m_{w} p+\left(2 m_{w}+6\right)\left(1+m_{w}\right) \sqrt{9 p^{2}-6\left(2 m_{w}+3\right) /\left(1+m_{w}\right)}\right]>0 } \\
& \text { for } p^{2}>p_{*}^{2}=\frac{8\left(1+m_{w}\right)\left(m_{w}+3\right)^{2}}{3\left(2+m_{w}\right)\left(2 m_{w}^{2}+9 m_{w}+6\right)} .
\end{aligned}
$$

A simple computation shows that $p_{*}^{2} \leq \frac{2\left(2 m_{w}+3\right)}{3\left(1+m_{w}\right)} \leq 2$, where $p^{2} \geq \frac{2\left(2 m_{w}+3\right)}{3\left(1+m_{w}\right)}$ is the existence condition from (3.41). Also it holds that for $p^{2} \geq 2$ the coefficient $a_{0}$ is positive for all values of $m_{w}$.

A computer test of the coefficient $a_{1}$ for $a_{w}=a_{w}^{-}$, shows that it is positive for $p^{2} \geq 2$. Thus, the solution with $a_{w}=a_{w}^{-}$is stable for $p^{2} \geq 2$. 


\section{Numerical results}

A direct numerical simulation of (1.2) was performed in order to check the validity of the lowdimensional model. We have used a pseudospectral code with time integration in Fourier space, periodic boundary conditions, Crank-Nicholson scheme for the linear operator and Adams-Bashforth scheme for the nonlinear one. The results of simulations for two values of the domain length are compared with the values predicted by the ODE-model in Figure 1. The results of a direct numerical simulation for a large value of $w$ are presented in Figure 2.

a)

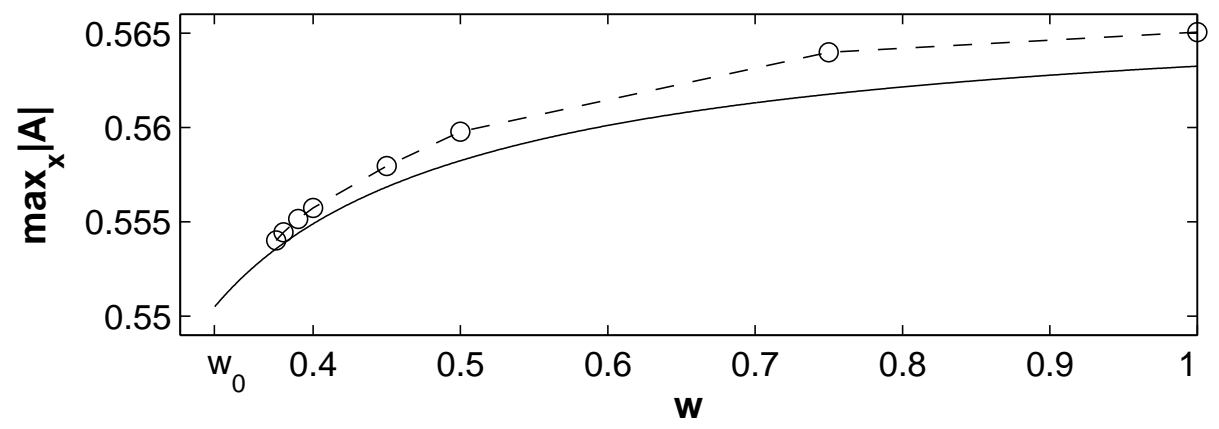

b)

c)

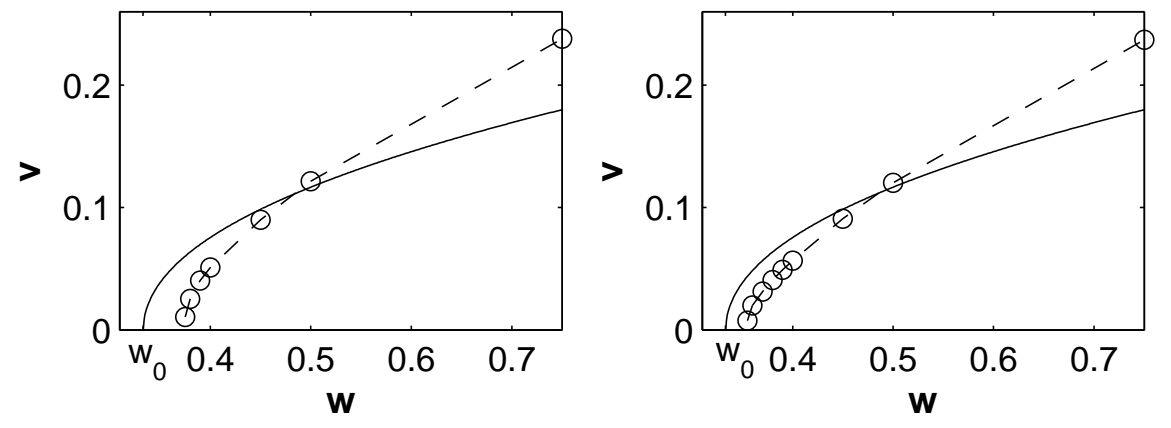

Figure 1: Bifurcation diagram for $m_{w}=1, p=2$ : a) dependence of maximum amplitude on $w$ for $L=20 \pi$; b) dependence of $\mathrm{v}$ on $w$ for $L=20 \pi$; c) dependence of $\mathrm{v}$ on $w$ for $L=40 \pi$. $w_{0}=1 / 3$. Solid lines: analytical results given by (3.26) and (3.29). Dashed lines: results of a direct numerical simulation of (1.2).

The theoretical value of $w_{0}$ given by (3.15) is derived in the limit $L \rightarrow \infty$, while numerical results are obtained for the finite values of the domain length $L$ (see Figure 1). For the maximum amplitude value $\max _{x}|A|$ it holds that three digits of analytical and numerical results coincide, see Figure 1(a). The comparison of the theoretical results and the results of the direct numerical simulation of (1.2) for the dependence of the velocity of the traveling wave $\mathrm{v}$ on the coupling parameter $w$ allows us to make the following conclusions: $(i)$ the theoretical prediction of $\mathrm{v}(w)$ is valid near the bifurcation point, that is, for relatively small values of $w$; and (ii) the approximation of numerical results by the analytical ones is improved with the growth of the domain length value, when 
the numerical value of $w_{0}$ approaches the theoretical value given by (2.23), see Figure 1(b),(c).

The explanation why the ODE-model fails to predict the dependence $\mathrm{v}(w)$ for large values of the coupling parameter $w$ is obvious from Figure 2. For large values of $w$ the traveling wave changes its shape [see Figure 2(b)], therefore the ansatz utilized in our ODE-model can not describe the actual dynamics of the traveling wave.

a)

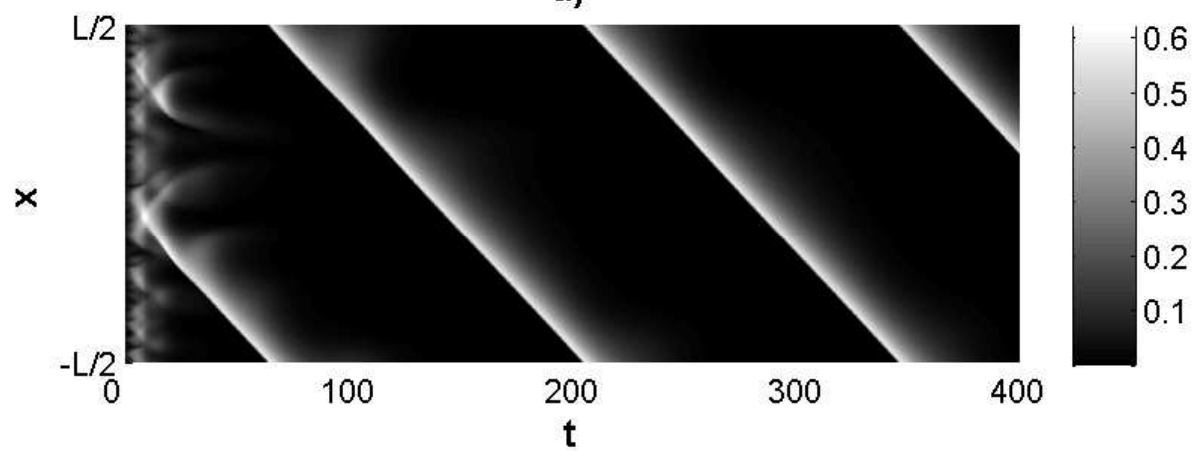

b)

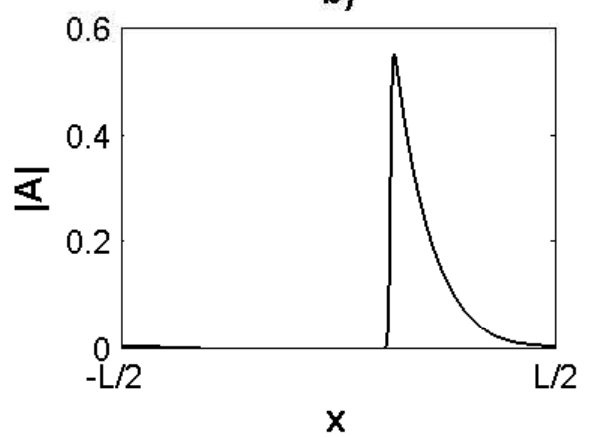

c)

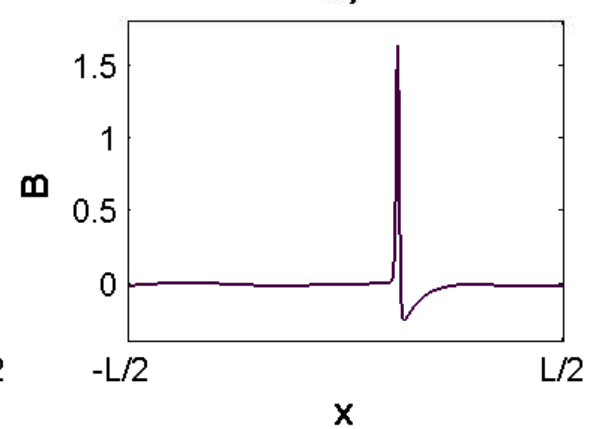

Figure 2: Dynamical regime for $L=80 \pi, p=2, m_{w}=1$ and $w=20$ : a) spatio-temporal plot of $|A|$; b) plot of $|A(x)|$; c) plot of $B(x)$.

\section{Acknowledgments}

The support of Israel Science Foundation (grant \# 812/06) and Minerva Center for Nonlinear Physics of Complex Systems is acknowledged. This work was inspired by valuable discussions with A.A. Golovin. 


\section{Appendix}

Derivation of (3.3). Assuming that $A, B, A_{x}$ and $B_{x}$ are functions of $b_{i}(t), i=1, \ldots, N$, and $x$, we find:

$$
\begin{aligned}
& -\frac{\partial<\mathcal{L}>}{\partial b_{i}}=-\int_{-L / 2}^{L / 2}\left[\frac{\partial \mathcal{L}}{\partial A} \frac{\partial A}{\partial b_{i}}+\frac{\partial \mathcal{L}}{\partial B} \frac{\partial B}{\partial b_{i}}+\frac{\partial \mathcal{L}}{\partial A_{x}} \frac{\partial A_{x}}{\partial b_{i}}+\frac{\partial \mathcal{L}}{\partial B_{x}} \frac{\partial B_{x}}{\partial b_{i}}\right] d x \\
& =\int_{-L / 2}^{L / 2}\left[\left(-\frac{\partial \mathcal{L}}{\partial A}\right) \frac{\partial A}{\partial b_{i}}+\left(-\frac{\partial \mathcal{L}}{\partial B}\right) \frac{\partial B}{\partial b_{i}}-\frac{\partial \mathcal{L}}{\partial A_{x}} \frac{\partial}{\partial x} \frac{\partial A}{\partial b_{i}}-\frac{\partial \mathcal{L}}{\partial B_{x}} \frac{\partial}{\partial x} \frac{\partial B}{\partial b_{i}}\right] d x \\
& =\int_{-L / 2}^{L / 2}\left[\left(Q_{1}-\frac{\partial}{\partial x} \frac{\partial \mathcal{L}}{\partial A_{x}}\right) \frac{\partial A}{\partial b_{i}}+\left(Q_{2}-\frac{\partial}{\partial x} \frac{\partial \mathcal{L}}{\partial B_{x}}\right) \frac{\partial B}{\partial b_{i}}-\frac{\partial \mathcal{L}}{\partial A_{x}} \frac{\partial}{\partial x} \frac{\partial A}{\partial b_{i}}-\frac{\partial \mathcal{L}}{\partial B_{x}} \frac{\partial}{\partial x} \frac{\partial B}{\partial b_{i}}\right] d x \\
& =\int_{-L / 2}^{L / 2}\left(Q_{1} \frac{\partial A}{\partial b_{i}}+Q_{2} \frac{\partial B}{\partial b_{i}}\right) d x-\underbrace{\int_{-L / 2}^{L / 2} \frac{\partial}{\partial x}\left[\frac{\partial \mathcal{L}}{\partial A_{x}} \frac{\partial A}{\partial b_{i}}+\frac{\partial \mathcal{L}}{\partial B_{x}} \frac{\partial B}{\partial b_{i}}\right] d x}_{=0} .
\end{aligned}
$$

\section{References}

[1] D. Casini, G. D’Alessandro, A. Politi. Soft turbulence in multimode lasers. Phys. Rev. A, 55 (1997), 751-760.

[2] S. Chávez Cerda, S.B. Cavalcanti, J.M. Hickmann. A variational approach of nonlinear dissipative pulse propagation. Eur. Phys. J. D, 1 (1998), 313-316.

[3] P. Coullet, S. Fauve. Propagative phase dynamics for systems with Galilean invariance. Phys. Rev. Lett., 55 (1985), 2857-2859.

[4] P. Coullet, G. Iooss. Instabilities of one-dimensional cellular patterns. Phys. Rev. Lett., 64 (1990), 866-869.

[5] S.M. Cox, P.C. Matthews. Instability and localisation of patterns due to a conserved quantity. Physica D, 175 (2003), 196-219.

[6] A.A. Golovin, S.H. Davis, P.W. Voorhees. Self-organization of quantum dots in epitaxially strained solid films. Phys. Rev. E, 68 (2003), 056203.

[7] A.A. Golovin, Y. Kanevsky, A.A. Nepomnyashchy. Feedback control of subcritical Turing instability with zero mode. Phys. Rev. E, 79 (2009), 046218. 
[8] A.A. Golovin, A.A. Nepomnyashchy. Feedback control of subcritical oscillatory instabilities. Phys. Rev. E, 73 (2006), 046212.

[9] A.A. Golovin, A.A. Nepomnyashchy, L.M. Pismen. Interaction between short-scale Marangoni convection and long-scale deformational instability. Phys. Fluids, 6 (1994), 34 47.

[10] A.A. Golovin, A.A. Nepomnyashchy, L.M. Pismen. Nonlinear evolution and secondary instabilities of Marangoni convection in a liquid-gas system with deformable interface. J. Fluid Mech., 341 (1997), 317-341.

[11] Y. Kanevsky, A.A. Nepomnyashchy. Stability and nonlinear dynamics of solitary waves generated by subcritical oscillatory instability under the action of feedback control. Phys. Rev. E, 76 (2007), 066305.

[12] Y. Kanevsky, A.A. Nepomnyashchy. Dynamics of solitary waves generated by subcritical instabiity under the action of delayed feedback control. Physica D, (2009), DOI: 10.1016/j.physd.2009.10.007.

[13] N. Komarova, A.C. Newell. Nonlinear dynamics of sandbanks and sandwaves. J. Fluid Mech., 415 (2000), 285-321.

[14] B.A. Malomed. Variational methods in nonlinear fiber optics and related fields. Progress in Optics, 43 (2002), 69-191.

[15] P.C. Matthews, S.M. Cox. One-dimensional pattern formation with Galilean invariance near a stationary bifurcation. Phys. Rev. E, 62 (2000), R1473-R1476.

[16] P.C. Matthews, S.M. Cox. Pattern formation with a conservation law. Nonlinearity, 13 (2000), 1293-1320.

[17] A.A. Nepomnyashchy, A.A. Golovin, V. Gubareva, V. Panfilov. Global feedback control of a long-wave morphological instability. Physica D, 199 (2004), 61-81.

[18] A.C. Newell, J.A. Whitehead. Finite amplitude convection. J. Fluid Mech., 38 (1969), 279303.

[19] B.Y. Rubinstein, A.A. Nepomnyashchy, A.A. Golovin. Stability of localized solutions in a subcritically unstable pattern-forming system under a global delayed control. Phys. Rev. E, 75 (2007), 046213.

[20] W. Schöpf, L. Kramer. Small-amplitude periodic and chaotic solutions of the complex Ginzburg-Landau equation for a subcritical bifurcation. Phys. Rev. Lett., 66 (1991), 23162319.

[21] M. Sheintuch, O. Nekhamkina. Analysis of front interaction and control in stationary patterns of reaction-diffusion systems. Phys. Rev. E, 63 (2001), 056120. 
[22] V. Skarka, N.B. Aleksić. Stability criterion for dissipative soliton solutions of the one-, two-, and three-dimensional complex cubic-quintic Ginzburg-Landau equations. Phys. Rev. Lett., 96 (2006), 013903.

[23] L.G. Stanton, A.A. Golovin. Global feedback control for pattern-forming systems. Phys. Rev. E, 76 (2007), 036210.

[24] E.N. Tsoy, A. Ankiewicz, N. Akhmediev. Dynamical models for dissipative localized waves of the complex Ginzburg-Landau equation. Phys. Rev. E, 73 (2006), 036621. 